\title{
On the sharpness of a three circles theorem for discrete harmonic functions
}

\author{
Gabor Lippner and Dan Mangoubi
}

\begin{abstract}
Any three circles theorem for discrete harmonic functions must contain an inherent error term. In this paper we find the sharp error term in an $L^{2}$-three circles theorem for harmonic functions defined in $\mathbb{Z}^{2}$. The proof is highly indirect due to combinatorial obstacles and cancellations phenomena. We exploit Newton interpolation methods and recursive arguments.
\end{abstract}

\section{Introduction}

Let $u$ be a discrete harmonic function defined in $\mathbb{Z}^{d}$. Let $\left(S_{n}\right)_{n=0}^{\infty}$ be the simple random walk starting at 0 and let $Q_{u}(n):=\mathbb{E}\left|u\left(S_{n}\right)\right|^{2}$ measure the $L^{2}$-growth of $u$. We recall that $Q_{u}$ is absolutely monotonic.

Theorem 1.1 ([LM15]). For all $k \in \mathbb{N}$

$$
Q_{u}^{(k)} \geq 0
$$

where $Q_{u}^{(k)}$ denotes the $k$ th forward discrete derivative.

As a formal corollary we concluded a three circles theorem with an inherent error term.

Corollary 1.2 ([LM15, Proof of Theorem 1.11]). Let $u: \mathbb{Z}^{d} \rightarrow \mathbb{C}$ be harmonic. Then

$$
\forall n \in \mathbb{N} \quad Q_{u}(2 n) \leq 2 \sqrt{Q_{u}(n) Q_{u}(4 n)}+2^{-\sqrt{n}} Q_{u}(4 n) .
$$


The estimate (11) holds for all absolutely monotonic functions $f: \mathbb{N} \rightarrow \mathbb{R}$ in place of $Q_{u}$. On the one hand, if we let $\beta>1 / 2$, we can always find absolutely monotonic functions which give rise to an error term larger than $2^{-n^{\beta}}$, e.g., $f(n)=\left(\begin{array}{l}n \\ k\end{array}\right)$ where $k$ is large (see Theorem 5.2). On the other hand, there exist absolutely monotonic functions, $f$, which satisfy $f(2 n) \leq \sqrt{f(n) f(4 n)}$ without any error term, as can be easily verified for $f(n)=\left(\begin{array}{c}n+k \\ k\end{array}\right)$. These two sequences of absolutely monotonic functions represent the two extreme behaviors with respect to the error term in (11). Thus, we are naturally led to ask where functions of the type $Q_{u}$ fall in between these two extremes.

In this paper we prove that, in fact, the error $2^{-\sqrt{n}}$ cannot be improved in Corollary 1.2 for $d=2$ in the following strong sense.

Theorem 1.3. For all $\varepsilon>0, A>0$ and $n_{0} \in \mathbb{N}$ there exists a harmonic function $u: \mathbb{Z}^{2} \rightarrow \mathbb{C}$ and $n>n_{0}$ such that

$$
Q_{u}(2 n)>A \sqrt{Q_{u}(n) Q_{u}(4 n)}+2^{-n^{1 / 2+\varepsilon}} Q_{u}(4 n) .
$$

We recall [LM15, Theorem 1.13] that for any given $A>0, \varepsilon>0$, by allowing the number of dimensions, $d$, to be large enough one can find a harmonic function on $u: \mathbb{Z}^{d} \rightarrow \mathbb{R}$ such that $Q_{u}$ satisfies (2). In light of this fact, the emphasis in the present article is on the sharpness of the error term in a given number of dimensions, and we resolve this question in two dimensions.

More generally, we believe that the theme of understanding the way the optimal error is affected by the properties of the underlying random walk may be of high interest as a link between analysis and geometry. Furthermore, this question is linked to the following broader question: What is the ensemble of absolutely monotonic functions which can be realized as $Q_{u}$ for some harmonic function $u$ on $\mathbb{Z}^{d}$ ? As suggested to us by Eugenia Malinnikova, it may be true also that the sharp error term we find is universal in the sense that different sharp discrete three circles theorems on $\mathbb{Z}^{d}$ have the same error term (see GM14]).

The route we take towards the proof of Theorem 1.3 is highly indirect due to combinatorial obstacles, seemingly very difficult to surpass, which we would otherwise face (see Section 1.1 for a more detailed explanation). In particular, after suggesting the candidate harmonic function $u$ with corresponding large error, we analyze $Q_{u}$ via its Newton expansion, and then compare $Q_{u}$ with a model absolutely monotonic function by proving rele- 
vant remainders estimates. Since the proof incorporates several independent ingredients we first describe the global picture in Section 1.1.

\section{$1.1 \quad$ Ideas and outline of proof}

Natural candidates for harmonic functions exhibiting corresponding large error terms are discrete analogs, $Z_{k}$, of the continuous harmonic functions defined by $z \mapsto z^{k} / k$ ! in $\mathbb{C}$. We have chosen to work with a discretization process having its roots in [Lov04] and adapted in [JLS14. The advantage of working with this discretization stems from the fact that it preserves harmonic functions. For short we denote $Q_{Z_{k}}$ by $Q_{k}$.

It has already been noted that $f_{k}(n)=\left(\begin{array}{l}n \\ k\end{array}\right)$ gives rise to an error arbitrarily close to $2^{-\sqrt{n}}$ when $k$ is large. One of the key observations in the proof of Theorem 1.3 is that there exists a one-parameter family of such sequences of essentially absolutely monotonic functions. This family is explicitly given by $f_{k, \alpha}(n):=\left(\begin{array}{c}n+\alpha k \\ k\end{array}\right)$ with $0 \leq \alpha<1 / 2$ (see Theorem 5.2). One may be surprised here as one expects that the optimal error for $f_{k, \alpha}$ drops continuously from $2^{-\sqrt{n}}$ to 0 as $\alpha$ goes from 0 to 1 .

Once we understand the behavior of the error term for the model family, $f_{k, \alpha}$, we aim to prove that $Q_{k}(n)$ is comparable with the $f_{k, 1 / 4}(n)$ in a certain range of $n$ 's. This comparison should be uniform in $k$ and, very importantly, be valid for $n$ 's much smaller than $k^{2}$, as this range is the source of the large error in the logarithmic convexity inequality with $f_{k, \alpha}$ (in fact, in the range $n>k^{2}$ all members of the family $\left(f_{k, \alpha}\right)_{0 \leq \alpha \leq 1}$ are comparable. In particular, $f_{k, \alpha}$ satisfies $f_{k, \alpha}(2 n) \leq 20 \sqrt{f_{k, \alpha}(n) f_{k, \alpha}(4 n)}$ with no error term for $n>k^{2}$, similarly to $f_{k, 1}$. See also [LM15, Theorem 1.12]).

As mentioned, the proof of the above comparison is highly indirect. The reason we are not able to approach the problem directly lies in complicated cancellation phenomena which occur in the expressions for $\left|Z_{k}\right|^{2}$. To get a feeling for this, try to prove that $\left|(x+i y)^{k}\right|^{2}=\left(x^{2}+y^{2}\right)^{k}$ just by expanding the parenthesis on the left hand side. This involves cancellation for alternating sums of binomial coefficients, the discrete analogues of which are involved to an extent that we are not able to keep track of anymore. We now describe in some detail the main ideas and steps in the proof of the discussed comparison. 
(a) We expand $Q_{k}$ as a Newton series.

$$
Q_{k}(n)=\sum_{j=0}^{k} a_{k, j}\left(\begin{array}{c}
n \\
k-j
\end{array}\right) .
$$

(b) We find a recursive behavior of $Q_{k}: a_{k, j} \approx 4^{-j} Q_{j}(k-j)$. This statement is made precise in Theorem 4.2.

(c) From the preceding step we can conclude (Theorem 4.3) that $a_{k, j}$ is a polynomial in $k$ of degree $j$ of which leading term is $(k / 4)^{j} / j$ !. At this stage it looks plausible that $Q_{k}$ behaves like $\left(\begin{array}{c}n+k / 4 \\ k\end{array}\right)$ since

$$
\left(\begin{array}{c}
n+k / 4 \\
k
\end{array}\right)=\sum_{j=0}^{k}\left(\begin{array}{c}
k / 4 \\
j
\end{array}\right)\left(\begin{array}{c}
n \\
k-j
\end{array}\right) .
$$

However, it is far from evident at this point whether this comparison is valid for any $n<k^{2}$ which is the relevant range.

(d) It turns out that in order to have sharp upper and lower bounds on $Q_{k}(n)$ in the range $n \gg k$ one needs to precisely understand $a_{k, j}$ only for $j \ll k$. Theorem 4.5 shows

$$
Q_{k}(n) \approx \sum_{j<k^{2} / n} a_{k, j}\left(\begin{array}{c}
n \\
k-j
\end{array}\right) .
$$

To prove this we need just rough upper and lower bounds on $a_{k, j}$ for all $k, j$. The lower bound we need is $a_{k, j} \geq 0$ which we get with no extra work (recall that we know this a priori from Theorem 1.1 or more directly from Step (b) ). The rough upper bound we need, Claim 4.4. follows from Step (b).

(e) We show (Corollary 8.3) that

$$
\left(\begin{array}{c}
n+k / 4 \\
k
\end{array}\right) \approx \sum_{j<k^{2} / n}\left(\begin{array}{c}
k / 4 \\
j
\end{array}\right)\left(\begin{array}{c}
n \\
k-j
\end{array}\right)
$$

(f) The comparison $Q_{k} \approx f_{k, 1 / 4}$ in a relevant range (Theorem 4.6) now follows from steps (c), (d) and (e). 
A guide to the reader. After reading the outline of the proof in Section 1.1, we suggest to concentrate first on the proof of the lower bound in Theorem 4.6. since all the main ideas are contained in that part of the proof, while it does not require Sections 4.3 and 7 . Although Theorem 1.1 stays in the background, we made an attempt to keep this paper independent of [LM15]. In particular, Theorem 1.1 for the special family $Q_{k}$ follows from Theorem 4.2. The only place where we do need three simple technical lemmas from [LM15] is in the proof of the recursive formula, Theorem 4.2, The basic notations and objects are introduced in Sections 2 and 3 , For convenience we give the list of dependencies for sections 4,8 : $7 \rightarrow 4.3 \rightarrow 4.4 \rightarrow 6$, $4.1 \rightarrow 4.2 \rightarrow 4.4,8 \rightarrow 4.3,8 \rightarrow 4.4$ and $5 \rightarrow 6$.

Acknowledgments. We would like to thank Eugenia Malinnikova for her influence on this work. Eugenia motivated us to look for the optimal error term, after finding together with Maru Guadie in GM14 a first formulation of a three circles theorem with an error, asking whether $2^{-\sqrt{n}}$ could be the optimal error. An important part of this work has been conducted while both authors were visiting the Rényi Institute. We wish to thank Miklós Abért and the Rényi Groups and Graphs research group for their generous support. The support of the BSF is gratefully acknowledged (grants nos. 2010214 and 2014108). The second author gratefully acknowledges the support of the ISF (grant no. $753 / 14$ ).

\section{Basic notations and definitions}

We fix notations and definitions we use throughout the paper.

Let $S=\{(0,1),(0,-1),(1,0),(-1,0)\}$ be the standard symmetric generating set for $\mathbb{Z}^{2}$.

Definition 2.1. For $u: \mathbb{Z}^{2} \rightarrow \mathbb{C}$ and $s \in S$ let

$$
\partial_{s} u(x):=u(x+s)-u(x) .
$$

We now define the Laplace operator acting on functions on $\mathbb{Z}^{2}$.

Definition 2.2. Let $u: \mathbb{Z}^{2} \rightarrow \mathbb{C}$.

$$
(\Delta u)(x):=\frac{1}{4} \sum_{s \in S}\left(\partial_{s} u\right)(x) .
$$


Definition 2.3. A function $u: \mathbb{Z}^{2} \rightarrow \mathbb{C}$ is called harmonic if $\Delta u=0$.

Let $\left(S_{n}\right)_{n=1}^{\infty}$ be the simple random walk on $\mathbb{Z}^{2}$ starting at 0 . We measure the $L^{2}$-growth of $u$ in terms of the random walk.

\section{Definition 2.4.}

$$
Q_{u}(n):=\mathbb{E}\left|u\left(S_{n}\right)\right|^{2} .
$$

A central role in our analysis is played also by the following family of sequences of binomial coefficients.

Definition 2.5. Let $0 \leq \alpha \leq 1$. Set $f_{k, \alpha}(n):=\left(\begin{array}{c}n+\alpha k \\ k\end{array}\right)$.

\section{$3 \quad$ A harmonic discretization of $z \mapsto z^{k} / k$ !}

In this section we define a sequence of discrete harmonic functions $Z_{k}$ on $\mathbb{Z}^{2}$, being our main object of study in order to prove Theorem 1.3.

We discretize $z \mapsto z^{k} / k$ ! following the recipe appearing in [JLS14].

\section{Definition 3.1.}

$$
F_{k}(x):=\left(\begin{array}{c}
x+\frac{k-1}{2} \\
k
\end{array}\right)=\frac{1}{k !} \prod_{j=0}^{k-1}\left(x+\frac{k-1}{2}-j\right) .
$$

$F_{k}$ can be thought of as a discretization of $x \rightarrow x^{k} / k$ !. Then, we define

\section{Definition 3.2.}

$$
Z_{k}(x, y):=\sum_{l=0}^{k} i^{l} F_{k-l}(x) F_{l}(y)
$$

Since $z^{k} / k !=\sum_{l=0}^{k} i^{l} \frac{x^{k-l}}{(k-l) !} \frac{y^{l}}{l !}$ we may think of $Z_{k}$ as discretizing $z \mapsto z^{k} / k !$. The special feature of this discretization is that it preserves harmonicity [JLS14, Lemma 2.1], i.e., $Z_{k}$ is a harmonic function.

Since the discretization process does not commute with a homothety we need the following variant of $Z_{k}$.

Definition 3.3. Let

$$
\tilde{Z}_{k}(x, y):=2^{k} Z_{k}\left(\frac{x}{2}, \frac{y}{2}\right)
$$

where by abuse of notations we assume $Z_{k}$ is defined also for half-integers.

Notation. For convenience we let

$$
Q_{k}:=Q_{Z_{k}}, \quad \tilde{Q}_{k}:=Q_{\tilde{Z}_{k}} .
$$




\section{Comparison of $Q_{k}$ with a model absolutely monotonic function}

In Section 4.1 we derive an approximate recursive formula for $Q_{k}$, leading us in Section 4.2 to the asymptotic behavior of the Newton coefficients $a_{k, j}$. In Section 4.3 it gives us a way to estimate $Q_{k}(n)$ in the range $n \gg k$ knowing the behavior of $Q_{j}(n)$ only for $j \ll k$. Finally, in Section 4.4 we are able to compare $Q_{k}$ with a model essentially absolutely monotonic function.

\subsection{The recursive nature of $Q_{k}$}

We recall that Newton's interpolation (see e.g. [CJ99, p. 473]) gives that for any function $f: \mathbb{N} \rightarrow \mathbb{R}$

$$
f(n)=\sum_{j=0}^{n} f^{(j)}(0)\left(\begin{array}{l}
n \\
j
\end{array}\right),
$$

where $f^{(j)}$ denotes the $j$ th forward discrete derivative of $f$.

Accordingly, we define

Definition 4.1. The Newton coefficients $a_{k, j}$ are defined by the identity

$$
\forall n \in \mathbb{N} \quad Q_{k}(n)=\sum_{j=0}^{k} a_{k, j}\left(\begin{array}{c}
n \\
k-j
\end{array}\right)
$$

In view of the above, $a_{k, j}=Q_{k}^{(k-j)}(0)$. The following theorem shows that $Q_{k}$ has an approximate recursive nature.

\section{Theorem 4.2.}

$$
a_{k, j}=4^{-j} \tilde{Q}_{j}(k-j)
$$

In particular, $a_{k, 0}=1$.

Proof. We recall [LM15, Lemma 2.4] that

$$
a_{k, j}=Q_{k}^{(k-j)}(0)=\Delta^{k-j}\left|Z_{k}\right|^{2}(0) .
$$

On the other hand, we know [LM15, Remark 2.3] that for any harmonic function $f: \mathbb{Z}^{2} \rightarrow \mathbb{C}$

$$
\Delta^{l}\left(|f|^{2}\right)(0)=4^{-l} \sum_{s_{1}, \ldots s_{l}}\left|\partial_{s_{1}} \ldots \partial_{s_{l}} f\right|^{2}(0)
$$


where all $s_{i}$ 's are in the symmetric generating set $S$. In order to substitute in (4) $f=Z_{k}$ we readily compute [LM15, Proposition 5.2]

$$
\partial_{s}\left(Z_{k}\right)(p)=Z_{k-1}\left(p+\frac{s}{2}\right) .
$$

Combining (3), (41) and (15) together we get

$$
\begin{aligned}
a_{k, j} & =4^{-(k-j)} \sum_{s_{1}, \ldots s_{k-j}}\left|\partial_{s_{1}} \ldots \partial_{s_{k-j}} Z_{k}\right|^{2}(0) \\
& =4^{-(k-j)} \sum_{s_{1}, \ldots s_{k-j}}\left|Z_{j}\left(\left(s_{1}+\ldots+s_{k-j}\right) / 2\right)\right|^{2} \\
& =4^{-(k-j)} \sum_{s_{1}, \ldots s_{k-j}}\left|2^{-j} \tilde{Z}_{j}\left(s_{1}+\ldots+s_{k-j}\right)\right|^{2}=4^{-j} \mathbb{E}\left|\tilde{Z}_{j}\left(S_{k-j}\right)\right|^{2}
\end{aligned}
$$

\subsection{Asymptotics of $a_{k, j}$ as a function of $k$}

From Theorem 4.2 we can obtain the asymptotics of $a_{k, j}$ as a function of $k$.

\section{Theorem 4.3.}

$$
a_{k, j}=\frac{1}{j !}\left(\frac{k}{4}\right)^{j}+P_{j-1}(k),
$$

where $P_{j-1}$ is a polynomial of degree $j-1$ at most.

Proof. First, let us see that $a_{k, j}$ is indeed a polynomial in $k$ of degree $j$. Momentarily, let us write $a_{k, j}=a_{j}(k-j)$ to emphasize the dependence on $k$. By Theorem 4.2

$$
a_{j}(m)=4^{-j} \tilde{Q}_{j}(m)=4^{-j} \mathbb{E}\left|\tilde{Z}_{j}\right|^{2}\left(S_{m}\right) .
$$

Consequently [LM15, Lemma 2.4],

$$
a_{j}^{(l)}(m)=4^{-j} \mathbb{E}\left(\Delta^{l}\left|\tilde{Z}_{j}\right|^{2}\right)\left(S_{m}\right) .
$$

Since $\Delta^{l}\left|\tilde{Z}_{j}\right|^{2}$ is a polynomial of degree $2 j-2 l$, we get that $a_{j}$ is a polynomial of degree $j$ of which leading coefficient is $\frac{1}{j !} 4^{-j} \Delta^{j}\left(\left|\tilde{Z}_{j}\right|^{2}\right)(0,0)$.

On the other hand, $\left|\tilde{Z}_{j}(x, y)\right|^{2}=\left(x^{2}+y^{2}\right)^{j} /(j !)^{2}+P_{2 j-1}(x, y)$, where $P_{2 j-1}$ is a polynomial of degree at most $2 j-1$. It is not difficult to check that the highest degree term of $\Delta\left(x^{2}+y^{2}\right)^{j}$ is $j^{2}\left(x^{2}+y^{2}\right)^{j-1}$ and inductively conclude the statement of the theorem. 


\subsection{A truncated Newton expansion for $Q_{k}$}

The crucial theorem 4.5 below shows that in order to have a grasp on $Q_{k}(n)$ in the range $n \gg k$ it is sufficient to understand the coefficients $a_{k, j}$ for a range of $j$ 's much smaller than $k$. To prove it we only need rough bounds on the coefficients $a_{k, j}$ for all $k, j$ which follow from Theorem 4.2. This section is required only for the upper bound in Theorem 4.6 and can be skipped on a first reading.

Claim 4.4. There exist $A_{1}>0, B_{1}>0$ such that

$$
\forall k \geq j \quad 0 \leq a_{k, j} \leq B_{1}\left(\begin{array}{c}
A_{1} k \\
j
\end{array}\right)
$$

Proof. We consider the expression for $a_{k, j}$ given in Theorem 4.2. The left hand side inequality is immediate (as is seen a priori from Theorem 1.1). For the right hand side inequality we see that we need to bound $\tilde{Q}_{j}$ from above. To that end we examine definition 2.4 and successively bound the objects involved in it. This is done in Section 17. We find the estimate in Proposition 7.4. If $k-j \geq j$ we conclude that $a_{k, j}<8(20 k)^{j} / j$ !, and if $k-j<j$, we use $(20 e)^{j} \leq(20 e k)^{j} / j$ ! for $k \geq j$ to deduce that $a_{k, j}<8(20 e k)^{j} / j$ !. Finally, recall that by a Stirling type estimate we can find two positive constants $c_{0}, c_{1}$ such that

$$
c_{0}\left(\frac{n}{e}\right)^{n} \sqrt{n} \leq n ! \leq c_{1}\left(\frac{n}{e}\right)^{n} \sqrt{n} .
$$

The preceding estimates immediately give

$$
a_{k, j} \leq 8 \frac{(20 e k)^{j}}{j !} \leq 8 \frac{c_{1}}{c_{0}}\left(\begin{array}{c}
20 e^{2} k \\
j
\end{array}\right)
$$

Theorem 4.5. There exist $A>5, B>1$ such that if $n>2 k$ then

$$
\sum_{j \leq 5 k^{2} / n} a_{k, j}\left(\begin{array}{c}
n \\
k-j
\end{array}\right) \leq Q_{k}(n) \leq B \sum_{j \leq A k^{2} / n} a_{k, j}\left(\begin{array}{c}
n \\
k-j
\end{array}\right) .
$$

Proof. The left hand side inequality follows from the lower bound $a_{k, j} \geq 0$ and Definition 4.1. To prove the right hand side inequality, let $A_{1}>0, B_{1}>0$ 
be as in Claim 4.4. Then, we can estimate the tail in Newton's expansion of $Q_{k}(n)$ as follows.

$$
\sum_{j>6 e A_{1} k^{2} / n} a_{k, j}\left(\begin{array}{c}
n \\
k-j
\end{array}\right) \leq B_{1} \sum_{j>6 e A_{1} k^{2} / n}\left(\begin{array}{c}
A_{1} k \\
j
\end{array}\right)\left(\begin{array}{c}
n \\
k-j
\end{array}\right) \stackrel{(*)}{\leq} \frac{B_{1}}{2}\left(\begin{array}{l}
n \\
k
\end{array}\right)=\frac{B_{1}}{2} a_{k, 0}\left(\begin{array}{l}
n \\
k
\end{array}\right),
$$

where inequality $(*)$ follows from the remainder estimate in Lemma 8.2. Combining this together with Definition 4.1 we conclude the desired estimate with $A=6 e A_{1}$ and $B=1+B_{1} / 2$.

\subsection{Comparison of $Q_{k}$ with $f_{k, 1 / 4}$}

We compare $Q_{k}(n)$ with $f_{k, 1 / 4}(n)$ (see Definition 2.5). It is crucial that this comparison be valid for a range of $n$ 's much smaller than $k^{2}$.

Theorem 4.6. There exists a function $\psi: \mathbb{N} \rightarrow[0, \infty)$, monotonically increasing to $\infty$, and $C>0$ such that for all $k \in \mathbb{N}$ and $n>k^{2} / \psi(k)$

$$
\frac{1}{4}\left(\begin{array}{c}
n+\frac{k}{4} \\
k
\end{array}\right) \leq Q_{k}(n) \leq C\left(\begin{array}{c}
n+\frac{k}{4} \\
k
\end{array}\right) .
$$

Proof. According to Theorem 4.3 there exists a monotonically increasing function $\phi: \mathbb{N} \rightarrow \mathbb{N}$ such that if $k>\phi(j)$ then

$$
\frac{1}{2}\left(\begin{array}{c}
k / 4 \\
j
\end{array}\right)<a_{k, j}<2\left(\begin{array}{c}
k / 4 \\
j
\end{array}\right) \text {. }
$$

To prove the upper bound on $Q_{k}$ let $A$ and $B$ be as in Theorem 4.5. Let $\psi=\left\lceil\phi^{-1} / A\right\rceil$. If $n>k^{2} / \psi(k)$ and $j \leq A k^{2} / n$ then $\phi(j)<k$. Also, since $A>5$ and $\phi(k) \geq k, \psi(k)<k / 2$. So, the assumption $n>k^{2} / \psi(k)$ implies $n>2 k$. Hence, by Theorem 4.5 and (6) we have

$$
Q_{k}(n) \leq 2 B \sum_{j \leq A k^{2} / n}\left(\begin{array}{c}
k / 4 \\
j
\end{array}\right)\left(\begin{array}{c}
n \\
k-j
\end{array}\right) \leq 2 B\left(\begin{array}{c}
n+k / 4 \\
k
\end{array}\right),
$$

where the last inequality follows from Lemma 8.1.

We now prove the lower bound on $Q_{k}$. If $n>k^{2} / \psi(k)$ and $j \leq 5 k^{2} / n$ then, again, $\phi(j)<k$ and $n>2 k$. Hence, by the very same Theorem 4.5 and (6)

$$
Q_{k}(n) \geq \frac{1}{2} \sum_{j \leq 5 k^{2} / n}\left(\begin{array}{c}
k / 4 \\
j
\end{array}\right)\left(\begin{array}{c}
n \\
k-j
\end{array}\right) \geq \frac{1}{4}\left(\begin{array}{c}
n+\frac{k}{4} \\
k
\end{array}\right)
$$

where the last inequality is due to Corollary 8.3 . 


\section{A model absolutely monotonic family}

We study the error term in a model family of essentially absolutely monotonic functions. We recall Definition 2.5 and check

Lemma 5.1. $f_{k, \alpha}$ is absolutely monotonic in the range $[k, \infty)$.

Proof. Observe that $f_{k, \alpha}^{(j)}(n)=f_{k-j, \alpha}(n+j \alpha) \geq 0$ for $n \geq k$.

We show that for $\alpha<1 / 2$ we have a saturation of the error term in the corresponding logarithmic convexity inequality.

Theorem 5.2. Let $\varepsilon>0, C>0$ and $0 \leq \alpha<1 / 2$. Then, for all $k \in \mathbb{N}$ large enough and $\delta>0$ small enough the inequality

$$
f_{k, \alpha}(2 n)>C \sqrt{f_{k, \alpha}(n) f_{k, \alpha}(4 n)}+2^{-n^{1 / 2+\varepsilon}} f_{k, \alpha}(4 n)
$$

holds for all $n \in\left[k^{2-\varepsilon}, \delta k^{2}\right]$.

Proof. We have for $k$ large enough and $n>k^{2-\varepsilon}$

$$
\begin{aligned}
\frac{f_{k, \alpha}(2 n)}{f_{k, \alpha}(4 n)} & =\prod_{j=0}^{k-1} \frac{2 n+\alpha k-j}{4 n+\alpha k-j}=\frac{1}{2^{k}} \prod_{j=0}^{k-1}\left(1-\frac{j-\alpha k}{4 n-j+\alpha k}\right) \\
& \geq \frac{1}{2^{k}} \prod_{\alpha k<j \leq k-1}\left(1-\frac{j-\alpha k}{4 n-j+\alpha k}\right) \geq \frac{1}{2^{k}}\left(1-\frac{k-\alpha k}{4 n-k+\alpha k}\right)^{k} \\
& \geq \frac{1}{2^{k}}\left(1-\frac{k}{3 n}\right)^{k} \geq \frac{1}{2^{k}} e^{-k^{2} /(2 n)} \geq 2^{-2 k} \geq 2^{-n^{1 / 2+\varepsilon}} .
\end{aligned}
$$

The preceding inequality holds for $0 \leq \alpha \leq 1$. On the other hand, for $\alpha<1 / 2$ we also have

$$
\begin{aligned}
\frac{f_{k, \alpha}(2 n)^{2}}{f_{k, \alpha}(n) f_{k, \alpha}(4 n)} & =\prod_{j=0}^{k-1} \frac{(2 n+\alpha k-j)^{2}}{(n+\alpha k-j)(4 n+\alpha k-j)} \\
& =\prod_{j=0}^{k-1}\left(1+\frac{n(j-\alpha k)}{(n+\alpha k-j)(4 n+\alpha k-j)}\right)=: \prod_{j=0}^{k-1} A_{j} \\
& =A_{\lceil\alpha k\rceil} \prod_{j=0}^{\lceil\alpha k\rceil-1} A_{j} \cdot A_{2\lceil\alpha k\rceil-j} \prod_{j=2\lceil\alpha k\rceil+1}^{k-1} A_{j} \geq \prod_{j=2\lceil\alpha k\rceil+1}^{k-1} A_{j}
\end{aligned}
$$


where the last inequality is true due to the following elementary lemma of which proof we omit.

Lemma 5.3. Let $n \in \mathbb{N}$ and for $|x|<n$ set $F(x)=1+\frac{n x}{(n-x)(4 n-x)}$. If $y+z \geq 0,|y|,|z|<n$ then $F(y) F(z) \geq 1$.

From here we proceed to obtain for $n<\delta k^{2}$

$$
\begin{aligned}
\prod_{j=2\lceil\alpha k\rceil+1}^{k-1} A_{j} & \geq \prod_{j=\frac{k}{2}+\lceil\alpha k\rceil}^{k-1} A_{j} \geq\left(1+\frac{n k / 2}{4 n^{2}}\right)^{k / 2-\lceil\alpha k\rceil} \geq\left(1+\frac{1}{8 \delta k}\right)^{k(1 / 2-\alpha) / 2} \\
& \geq e^{(1-2 \alpha) /(40 \delta)}>C
\end{aligned}
$$

\section{Optimality of the error: Proof of Theo- rem 1.3}

We can now combine Theorem 4.6 and the study of the error term for the model function $f_{k, 1 / 4}$ in Section 5 to conclude that the error term in Corollary 1.2 is optimal in two dimensions.

Proof of Theorem 1.3. Fix $\varepsilon_{1}, C_{1}>0$ and let $\alpha=1 / 4$. Let $k_{0}=k\left(\varepsilon_{1}, C_{1}, \alpha\right)$ and $\delta=\delta\left(\varepsilon_{1}, C_{1}, \alpha\right)$ be as in Theorem 5.2. Let $\psi$ be as in Theorem 4.6. Let $k_{1} \in \mathbb{N}$ be large enough so that $k_{1}^{2} / \psi\left(k_{1}\right)<\delta k_{1}^{2}$ and $k_{1}^{2-\varepsilon_{1}}<\delta k_{1}^{2}$. Then, for $n, 2 n, 4 n \in\left[k_{1}^{2} / \psi\left(k_{1}\right), \delta k_{1}^{2}\right] \cap\left[k_{1}^{2-\varepsilon_{1}}, \delta k_{1}^{2}\right]$

$$
\begin{gathered}
Q_{k}(2 n) \stackrel{\operatorname{Th} 4.6}{\geq} \frac{1}{4} f_{k, 1 / 4}(2 n) \stackrel{\operatorname{Th} 5.2}{\geq} \frac{C_{1}}{4} \sqrt{f_{k, 1 / 4}(n) f_{k, 1 / 4}(4 n)}+\frac{1}{4} 2^{-n^{1 / 2+\varepsilon_{1}}} f_{k, 1 / 4}(4 n) \\
\stackrel{\operatorname{Th} 4.6}{\geq} C_{1} \tilde{C}_{1} \sqrt{Q_{k}(n) Q_{k}(4 n)}+\tilde{C}_{2} 2^{-n^{1 / 2+\varepsilon_{1}}} Q_{k}(4 n) .
\end{gathered}
$$

Hence, if we let $C_{1}=C / \tilde{C}_{1}$ and $\varepsilon_{1}=\varepsilon / 2$, we can take $k_{1}$ sufficiently large such that $\tilde{C}_{2} 2^{-n^{1 / 2+\varepsilon_{1}}}>2^{-n^{1 / 2+\varepsilon}}$ for $n>k_{1}^{2} / \psi\left(k_{1}\right)$ and then the stated inequality holds. 


\section{A rough upper bound on $\tilde{Q}_{k}$}

The goal of this section to prove the rough upper bound on $\tilde{Q}_{k}$ in Proposition 7.4. This section stands independent of all other sections and is required for the proof of the upper bound in Claim 4.4.

\subsection{A bound on $F_{k}$}

\section{Lemma 7.1.}

$$
F_{j}(x)^{2} \leq \frac{x^{2 j}}{j !^{2}}+1
$$

Proof. By symmetry we may assume $x \geq 0$. Observe that for $x \geq(j-1) / 2$ each factor in Definition 3.1 is nonnegative and obviously bounded by $x$. Hence $F_{j}(x) \leq x^{j} / j$ ! in this case.

Else, suppose $0 \leq x<(j-1) / 2$. Let $m=\left\lfloor\frac{j+1}{2}-x\right\rfloor$, and denote by $\{x\}=x-\lfloor x\rfloor$ the fractional part of $x$. Observe that

$$
\left|F_{j}(x)\right|=\frac{1}{j !} \prod_{l=0}^{m-1}(\{x\}+l)(l+1-\{x\}) \prod_{l=m}^{j-m-1}(\{x\}+l) .
$$

Hence,

$$
F_{j}(x)^{2} \leq \frac{1}{(j !)^{2}} \prod_{l=1}^{m-1}(l+1)^{4} \prod_{l=m}^{j-m-1}(l+1)^{2}=\frac{1}{\left(\begin{array}{c}
j \\
m
\end{array}\right)^{2}} \leq 1
$$

\subsection{Bounding $\tilde{Z}_{k}$}

\section{Proposition 7.2.}

$$
\left|\tilde{Z}_{k}(x, y)\right|^{2} \leq 3 \cdot 20^{k} \sum_{l=0}^{k} \frac{x^{2 l}+y^{2 l}}{4^{l} l !^{2}}
$$

Proof. We apply Lemma 7.1 to bound $Z_{k}(x, y)$.

$$
\left|Z_{k}(x, y)\right|^{2} \leq(k+1) \sum_{l=0}^{k} F_{k-l}(x)^{2} F_{l}(y)^{2}
$$




$$
\begin{aligned}
& \leq(k+1) \sum_{l=0}^{k}\left(\frac{x^{2 k-2 l}}{(k-l) !^{2}}+1\right)\left(\frac{y^{2 l}}{l !^{2}}+1\right) \\
& =(k+1) \sum_{l=0}^{k} \frac{x^{2 k-2 l} y^{2 l}}{(k-l) !^{2} l !^{2}}+(k+1) \sum_{l=0}^{k} \frac{x^{2 l}+y^{2 l}}{l !^{2}}+(k+1)^{2} \\
\text { (by Young's ineq.) } \leq & \frac{k+1}{k} \sum_{l=0}^{k} \frac{(k-l) x^{2 k}+l y^{2 k}}{(k-l) !^{2} l !^{2}}+2(k+1)^{2} \sum_{l=0}^{k} \frac{x^{2 l}+y^{2 l}}{l !^{2}} \\
& =\frac{k+1}{k} \sum_{l=0}^{k} \frac{l\left(x^{2 k}+y^{2 k}\right)}{l !^{2}(k-l) !^{2}}+2(k+1)^{2} \sum_{l=0}^{k} \frac{x^{2 l}+y^{2 l}}{l !^{2}} \\
& \leq(k+1) \frac{x^{2 k}+y^{2 k} k}{(k) !^{2}}(k)_{l=0}^{2}+2(k+1)^{2} \sum_{l=0}^{k} \frac{x^{2 l}+y^{2 l}}{l !^{2}} \\
& =(k+1)\left(\begin{array}{c}
2 k \\
k
\end{array}\right) \frac{x^{2 k}+y^{2 k}}{(k) !^{2}}+2(k+1)^{2} \sum_{l=0}^{k} \frac{x^{2 l}+y^{2 l}}{l !^{2}} \\
& \leq 3 \cdot 8^{k} \sum_{l=0}^{k} \frac{x^{2 l}+y^{2 l}}{l !^{2}} \cdot
\end{aligned}
$$

It remains to recall Definition 3.3 to get the desired estimate.

\subsection{Bounding $\tilde{Q}_{k}$}

We start with a standard random walk estimate.

\section{Lemma 7.3.}

$$
\mathbb{E} x^{2 k}\left(S_{n}\right) \leq \frac{(2 k) !}{4^{k} k !} n^{k}
$$

Proof. $X_{n}:=x\left(S_{n}\right)$ is a one dimensional lazy random walk of which generating function is $\varphi_{n}(t):=\mathbb{E} e^{t X_{n}}=\cosh ^{2 n}(t / 2)$. We can express its $2 k$ th moment as $\mathbb{E} X_{n}^{2 k}=\varphi_{n}^{(2 k)}(0)$. A direct calculation yields

$$
\varphi_{n}^{(2 k)}(0)=4^{-k} \sum_{j_{1}+\ldots+j_{2 n}=k}\left(\begin{array}{c}
2 k \\
2 j_{1}, 2 j_{2}, \ldots, 2 j_{2 n}
\end{array}\right)
$$


The preceding sum expression is the number of ways partitioning $2 k$ distinct objects into $2 n$ distinct drawers, where each drawer contains an even number of objects. Since each such partition can be obtained by first pairing the $2 k$ objects and then partitioning $k$ pairs into $2 n$ distinct drawers we get that the last sum is at most $\frac{(2 k) !}{2^{k} k !}(2 n)^{k}$.

Taking expectations in Proposition 7.2 and applying Lemma 7.3, we obtain

\section{Proposition 7.4.}

$$
\tilde{Q}_{k}(n)<8 \cdot \begin{cases}(20 n)^{k} / k ! & , \text { if } n \geq k \\ (20 e)^{k} & , \text { if } n<k\end{cases}
$$

Proof. We see from Proposition 7.2 and Lemma 7.3 that

$$
\tilde{Q}_{k}(n) \leq 6 \cdot 20^{k} \sum_{l=0}^{k} \frac{(2 l) !}{4^{2 l} l !^{3}} n^{l} \leq 6 \cdot 20^{k} \sum_{l=0}^{k} \frac{n^{l}}{4^{l} l !}
$$

Observe that if $n \geq k$ then $\frac{n^{l}}{l !} \leq \frac{n^{k}}{k !}$. Hence, in this case

$$
\tilde{Q}_{k}(n) \leq 6 \frac{(20 n)^{k}}{k !} \sum_{l=0}^{\infty} 4^{-l}=8 \frac{(20 n)^{k}}{k !} .
$$

If $n<k$ then $n^{l} / l !<k^{l} / l ! \leq k^{k} / k$ !. So, we obtain from (77) and the estimate $k ! \geq(k / e)^{k}$ that

$$
\tilde{Q}_{k}(n) \leq 6 \frac{(20 k)^{k}}{k !} \leq 6(20 e)^{k}
$$

\section{A Newton remainder estimate for $\left(\begin{array}{c}a+b \\ k\end{array}\right)$}

In this section we analyze the remainder in Newton's expansion for $\left(\begin{array}{c}a+b \\ k\end{array}\right)$.

It is not difficult to show

Lemma 8.1 (Vandermonde's identity).

$$
\left(\begin{array}{c}
a+b \\
k
\end{array}\right)=\sum_{j=0}^{k}\left(\begin{array}{c}
a \\
k-j
\end{array}\right)\left(\begin{array}{l}
b \\
j
\end{array}\right) .
$$


Proof. For nonnegative integers $a, b$ the identity follows from combinatorial interpretation of the binomial coefficients or by induction on $a$. Then, due to the polynomial nature of the expressions involved, it is true for all real numbers $a, b$.

The next Lemma estimates the remainder of the above expansion.

Lemma 8.2. Let $a \geq 2 k$. Then,

$$
\sum_{j>6 e k b / a}\left(\begin{array}{c}
a \\
k-j
\end{array}\right)\left(\begin{array}{l}
b \\
j
\end{array}\right) \leq \frac{1}{2}\left(\begin{array}{l}
a \\
k
\end{array}\right) .
$$

Proof.

$$
\begin{aligned}
\left(\begin{array}{c}
a \\
k-j
\end{array}\right)\left(\begin{array}{l}
b \\
j
\end{array}\right) & =\left(\begin{array}{l}
a \\
k
\end{array}\right) \frac{\left(\begin{array}{c}
k \\
j
\end{array}\right)\left(\begin{array}{l}
b \\
j
\end{array}\right)}{\left(\begin{array}{c}
a-k+j \\
j
\end{array}\right)} \leq\left(\begin{array}{l}
a \\
k
\end{array}\right) \frac{1}{j !} \frac{k^{j} b^{j}}{(a-k)^{j}} \\
& \leq\left(\begin{array}{l}
a \\
k
\end{array}\right) \frac{k^{j} b^{j} e^{j}}{j^{j}(a-k)^{j}} \leq\left(\begin{array}{l}
a \\
k
\end{array}\right)\left(\frac{2 e k b}{a j}\right)^{j}
\end{aligned}
$$

Hence,

$$
\sum_{j>6 e k b / a}\left(\begin{array}{c}
a \\
k-j
\end{array}\right)\left(\begin{array}{l}
b \\
j
\end{array}\right) \leq\left(\begin{array}{l}
a \\
k
\end{array}\right) \sum_{j=1}^{\infty} 3^{-j}=\frac{1}{2}\left(\begin{array}{l}
a \\
k
\end{array}\right)
$$

Corollary 8.3. Let $a \geq 2 k$. Then

$$
\sum_{j \leq 6 e k b / a}\left(\begin{array}{c}
a \\
k-j
\end{array}\right)\left(\begin{array}{l}
b \\
j
\end{array}\right) \geq \frac{1}{2}\left(\begin{array}{c}
a+b \\
k
\end{array}\right) .
$$

Proof. From Lemmas 8.1 and 8.2 we know that

$$
\sum_{j \leq 6 e k b / a}\left(\begin{array}{c}
a \\
k-j
\end{array}\right)\left(\begin{array}{l}
b \\
j
\end{array}\right)>\left(\begin{array}{c}
a+b \\
k
\end{array}\right)-\frac{1}{2}\left(\begin{array}{l}
a \\
k
\end{array}\right) \geq \frac{1}{2}\left(\begin{array}{c}
a+b \\
k
\end{array}\right) .
$$




\section{References}

[CJ99] R. Courant and F. John, Introduction to calculus and analysis. Vol. I, Classics in Mathematics, Springer-Verlag, Berlin, 1999. Reprint of the 1989 edition.

[JLS14] D. Jerison, L. Levine, and S. Sheffield, Internal DLA and the Gaussian free field, Duke Math. J. 163 (2014), no. 2, 267-308.

[GM14] M. Guadie and E. Malinnikova, On three balls theorem for discrete harmonic functions, Comput. Methods Funct. Theory 14 (2014), no. 4, 721-734.

[LM15] G. Lippner and D. Mangoubi, Harmonic functions on the lattice: Absolute monotonicity and propagation of smallness, Duke Math. J. 164 (2015), no. 13, 25772595.

[Lov04] L. Lovász, Discrete analytic functions: an exposition, Surveys in differential geometry. Vol. IX, Surv. Differ. Geom., IX, Int. Press, Somerville, MA, 2004, pp. 241273.

Gabor Lippner,

Department of Mathematics, Northeastern University, Boston, MA, USA;

g. lippner@neu.edu

Dan Mangoubi,

Einstein Institute of Mathematics, Hebrew University, Jerusalem, Israel;

mangoubi@math.huji.ac.il 